Korean Journal of Parasitology

Vol. 45, No. 2: 77-85, June 2007

\title{
Imaging diagnosis of clonorchiasis
}

\author{
Dongil $\mathrm{CHOI}^{1)}$ and Sung-Tae $\mathrm{HONG}^{2) *}$ \\ ${ }^{1)}$ Department of Radiology, Samsung Medical Center Sungkyunkwan University School of Medicine, Seoul 135-710, \\ ${ }^{2)}$ Department of Parasitology and Tropical Medicine, Seoul National University College of Medicine, Seoul 110-799, Korea
}

\begin{abstract}
Among several diagnostic tools for clonorchiasis (Clonorchis sinensis infection), radiologic examinations are commonly used in clinical practices. During the 2 past decades, many reports regarding imaging findings of clonorchiasis were introduced. The basic imaging finding of clonorchiasis is diffuse dilatation of the peripheral intrahepatic bile ducts, without dilation of the large intrahepatic or extrahepatic bile ducts. By this finding, however, active clonorchiasis cannot be differentiated from cured infection. Some recent radiologic studies suggested specific findings of active clonorchiasis. Besides direct demonstration of worms, increased periductal echogenicity on sonography and periductal enhancement on dynamic contrast-enhanced CT or MR imaging possibly represent active clonorchiasis. Those images of the liver clonorchiasis are known to be correlated with worm burdens (EPG counts) in their frequency and also severity. The images of cholangiocarcinoma associated with clonorchiasis show both the tumor with obstruction images and diffuse dilatation of the peripheral intrahepatic bile ducts. Radiological images can be a good practical alternative diagnostic method of clonorchiasis.
\end{abstract}

Key words: Clonorchis sinensis, clonorchiasis, cholagiocarcinoma, imaging diagnosis

\section{INTRODUCTION}

Detection of eggs in stool can lead to a definite diagnosis of Clonorchis sinensis infection (clonorchiasis), thus, clonorchiasis has been principally diagnosed by stool microscopic examination. There are several methods of stool microscopic examination, but the Kato-Katz method is regarded as the best one for clonorchiasis (Hong et al., 2003). However, the routine stool microscopic examinations usually performed in hospitals (without a specialized laboratory for parasites) are insufficient to detect a few $C$. sinensis eggs in the stool of patients with a light infection. Furthermore, collection of stools is becoming rather difficult due to low compliance of residents. In this

- Received 16 March 2007, accepted after revision 9 May 2007.

*Corresponding author (e-mail: hst@snu.ac.kr) context, several other diagnostic tools such as skin tests, serologic tests and radiologic examinations have been introduced. The skin test is known to be sensitive but not specific, and enzyme-linked immunosorbent assay (ELISA) is widely used for serodiagnosis with moderate sensitivity and specificity but cross reactions occur with other parasitic fluke infections (Chen et al., 1994; Hong, 2003).

As a majority of physicians have little interest and knowledge in clonorchiasis, neither of stool examination, skin test, and ELISA for clonorchiasis is recommended as a routine examination for patients with vague epigastric pain or non-specific symptoms of the digestive tract even in endemic areas. Practically, clonorchiasis is commonly diagnosed during sonographic examination of the abdomen mainly for other purposes.

At present, clonorchiasis is still endemic along sev- 
eral rivers and about 1.3 million infected people are estimated over the country in Korea (KAHP, 2004). It must be one major health problem in endemic areas, which should be of priority. The health authority and local governments in endemic areas are trying to find out infected residents and to treat them with praziquantel, but their trials are not so effective because its diagnosis is rather difficult.

In the present article, we reviewed imaging findings of clonorchiasis, including cholangiography, sonography, computed tomography (CT), and magnetic resonance (MR) imaging. We tried to summarize imaging findings and suggest guidelines for imaging diagnosis of clonorchiasis.

\section{CHOLANGIOGRAPHIC FINDINGS OF CLONORCHIASIS}

The principle imaging finding of clonorchiasis is diffuse dilatation of the peripheral intrahepatic bile ducts (Lim, 1990). However, the larger intrahepatic and extrahepatic bile ducts are not dilated or minimally dilated. The predominant dilatation of the peripheral intrahepatic ducts is due to the adult worms residing in the peripheral small bile ducts. Compared with normal subjects, many intrahepatic bile ducts are observed on cholangiography in patients with clonorchiasis due to the dilated peripheral intrahepatic bile ducts (Fig. 1A and B). Lim et al. (1992) reported "too many intrahepatic ducts" sign in 76\% (22 of 29) of patients with clonorchiasis on direct cholangiography.

The presence of the fluke bodies in the peripheral bile ducts can be demonstrated on a cholangiogram (Choi et al., 1984; Lim et al., 1992). Cholangiography shows oval, elliptical, or filamentous, small filling defects within the dilated peripheral intrahepatic bile ducts, representing the flukes (Fig. 1) (Lim et al., 1992). It is a pathognomonic finding on direct cholangiography, but the defects can be obscured by the contrast medium. The filling defects by the flukes are usually small and irregular, with a uniform size on direct cholangiography, which are easily differentiated from stones (Fig. 1) (Choi et al., 1984; Lim et al.,
1992; Jeong et al., 2004). Direct cholangiogram is obtained with an injection of iodine contrast material via catheter during either endoscopy or percutaneous biliary drainage (Fig. 1). Recently, non-invasive cholangiograms can be made by using MR imaging (Fig. 1) (Barish and Soto, 1997).

\section{SONOGRAPHIC FINDINGS OF CLONORCHIASIS}

Sonographic findings of diffuse dilatation of the intrahepatic bile ducts and increased periductal echogenicity are known to well reflect pathological changes associated with clonorchiasis (Fig. 2) (Lim et al., 1989; Hong et al., 1994). Sonography is considered a good tool for the diagnosis of clonorchiasis, particularly in cases with a moderate or heavy burden of worms. According to a prior study in Korea, sonography showed low sensitivity and low specificity in the diagnosis of active clonorchiasis (Hong et al., 1998). The study suggested that the low sensitivity was due to a light worm burden and that the low specificity was due to residual pathology after cure.

In a recent study conducted by us in China, increased periductal echogenicity and floating echogenic foci in the gallbladder were proposed as the 2 most significant sonographic findings of active clonorchiasis (Choi et al., 2004). Increased periductal echogenicity represents a thickening of the ductal wall according to mucosal hyperplasia and periductal fibrosis, and the floating echogenic foci indicate worms or desquamated material in the gallbladder (Figs. 2A, B, C) (Lim et al., 1989; Hong et al., 1994; Choi et al., 1999). In our study in China, increased periductal echogenicity showed a sensitivity and specificity for active clonorchiasis of $35 \%$ and $91 \%$, respectively (Choi et al., 2004). Floating echogenic foci in the gallbladder had a sensitivity and specificity of $28 \%$ and $94 \%$, respectively. However, floating echogenic foci within the gallbladder have been rarely documented in Korea (Lim et al., 1989; Lim, 1990).

In the past literature, diffuse dilatation of the intrahepatic bile ducts on sonographic examination has been considered a hallmark of clonorchiasis (Lim et 

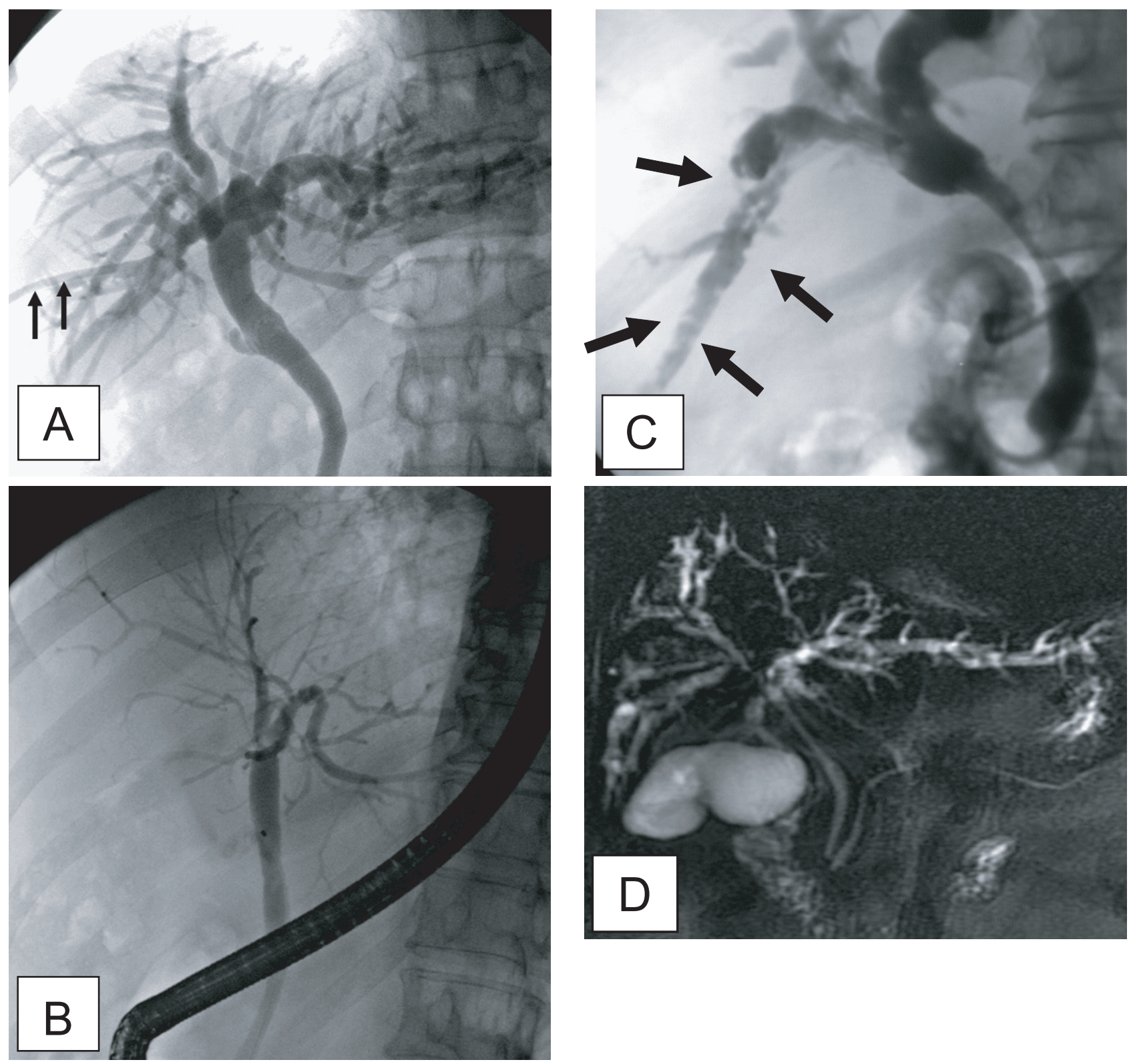

Fig. 1. Cholangiograms of clonorchiasis showing "too many intrahepatic ducts" signs. A. By percutaneous biliary drainage catheter (arrows) in a 58-year-old man, showing diffuse and uniform dilatation of the peripheral intrahepatic bile ducts. B. Endoscopic retrograde cholangiography in a 70-year-old man, with diffuse dilatation of the peripheral intrahepatic bile ducts, but no dilatation of the extrahepatic bile duct. C. Multiple, oval, small filling defects (arrows) within the dilated peripheral intrahepatic bile ducts, representing the flukes in a 55-year-old man. D. Magnetic resonance (MR) cholangiography in a 70-year-old man with diffuse dilatation of the peripheral intrahepatic bile ducts.

al., 1989; Lim, 1990; Hong et al., 1994). Diffuse dilatation of the intrahepatic bile ducts was found in an about half (49-52\%) of the control subjects in either present endemic area or past endemic area (Fig. 2D) (Hong et al., 1998; Choi et al., 2004). Thus, patients only with a dilatation of the intrahepatic bile ducts detected by sonography cannot be considered candidates for praziquantel treatment (Chen et al., 1994; Hong et al., 1998; Choi et al., 1999). In an experimental study, the histopathological specimens at 18 mo after treatment with praziquantel showed that periductal inflammation has almost resolved but moderate 

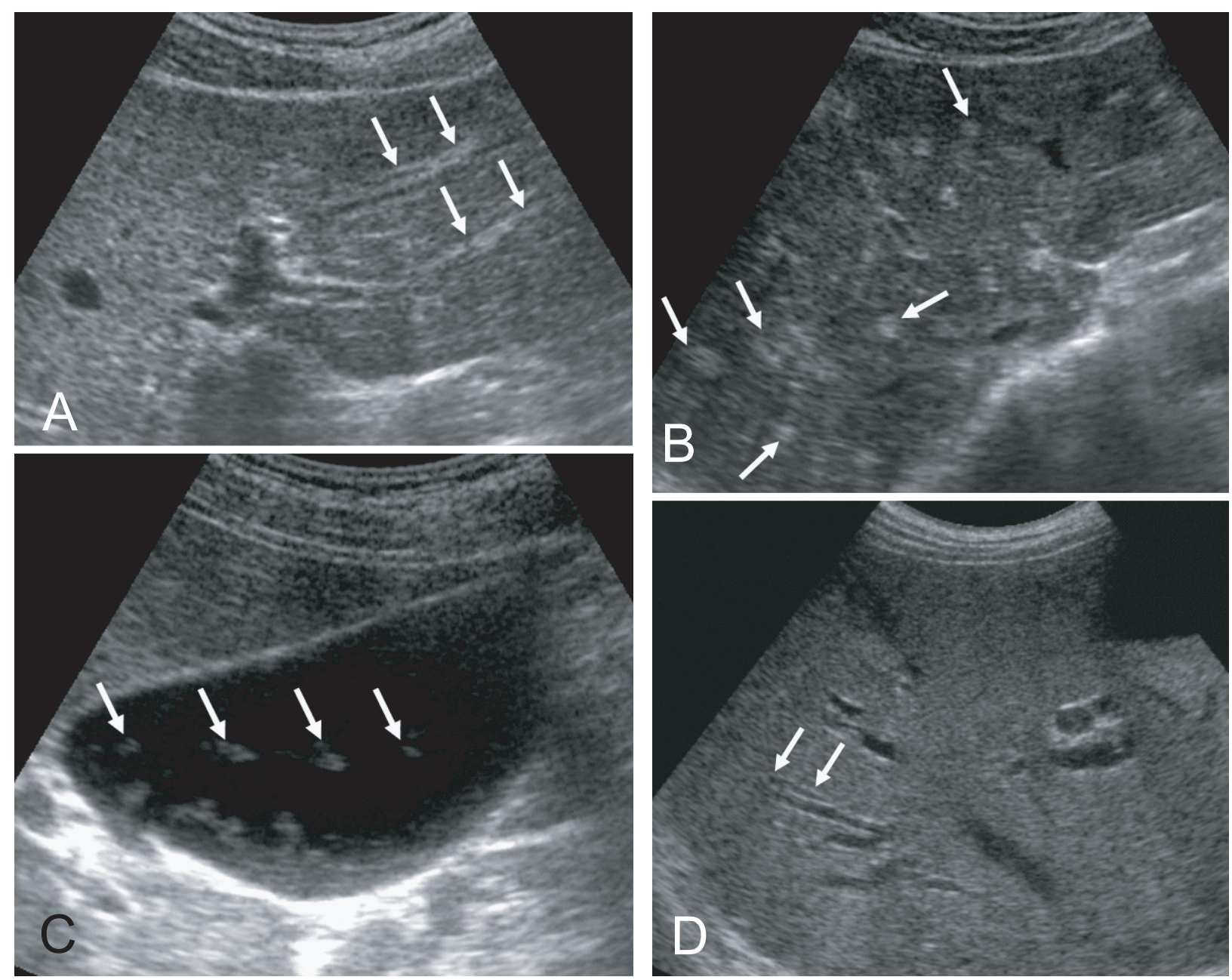

Fig. 2. Liver sonograms of clonorchiasis. A. In a 57-year-old man, transverse scan of the left hepatic lobe, showing mild dilatation of the intrahepatic bile ducts (arrows). B. In a 56-year-old man with the increased periductal echogenicity (arrows) along the dilated intrahepatic bile ducts. C. Oblique scan of the gallbladder of a heavily - infected Chinese man with several floating echogenic foci (arrows), which probably indicate worms or desquamated materials. D. Mild dilatation of the intrahepatic bile ducts in the central liver of a cured 60-year-old man.

dilatation of the intrahepatic ducts and mucosal hyperplasia persisted (Choi et al., 1999). The periductal fibrosis minimally resolved.

Although sonography is used as one of the screening diagnostic tools for clonorchiasis in endemic areas, sonography is a basically operator-dependent modality. Unless specific information is offered, many physicians and even radiologists cannot aware subtle changes of the intrahepatic bile ducts. It leads to low sensitivity to diagnose clonorchiasis, particularly in patients with light infection. Practically it is very difficult to make a correct diagnosis of lightly infected clonorchiasis by any method, even by stool examina- tion (Hong et al., 2003). Choi et al. (2005) recorded a significant correlation of the sonographic findings with egg counts. The correlation was significant by both frequency and grade of the findings. In other words, the more the flukes were, the more and severer were the findings.

\section{CT FINDINGS OF CLONORCHIASIS}

The CT finding of clonorchiasis is diffuse dilatation of the peripheral intrahepatic bile ducts, without dilation of the larger bile ducts or extrahepatic ducts (Fig. $3 \mathrm{~A})$. This finding is known to be the pathognomonic 

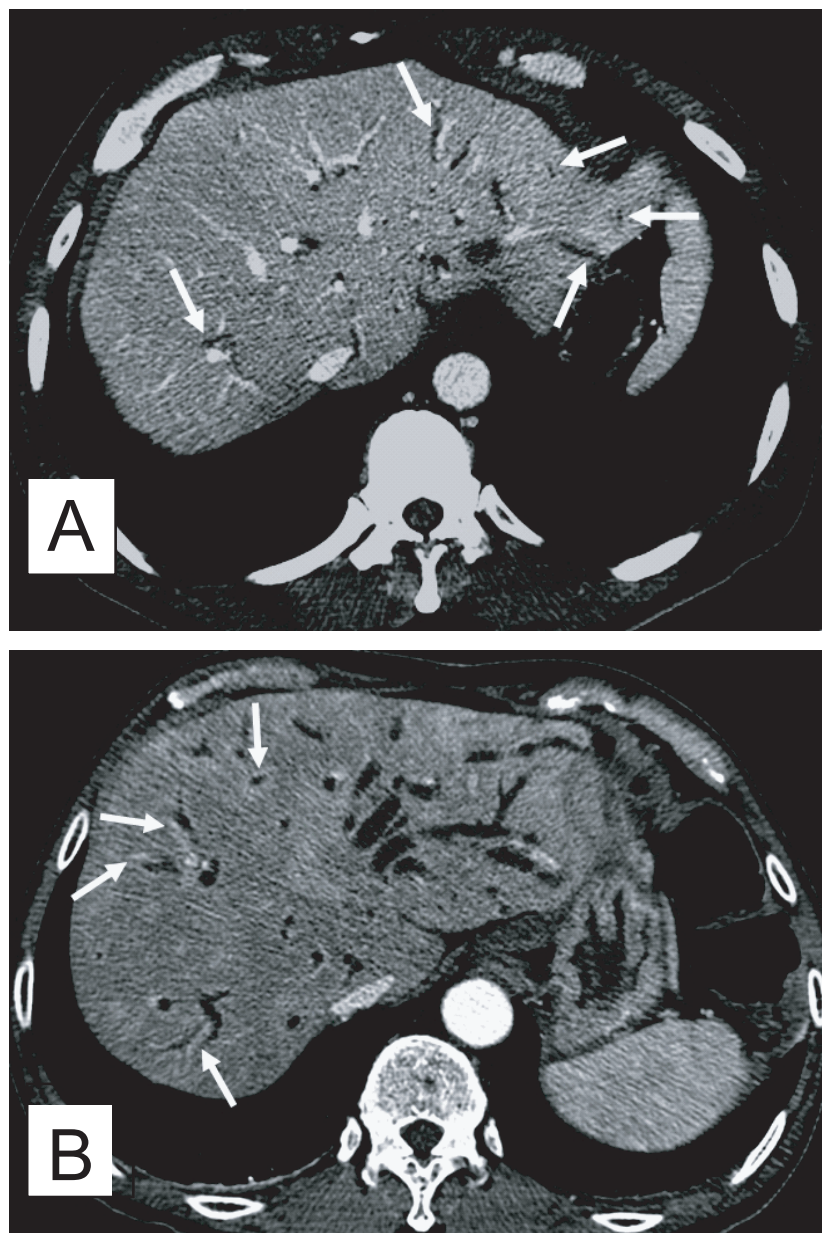

Fig. 3. Contrast-enhanced CT findings of the liver in clonorchiasis patients. A. A 52-year-old man, who underwent liver transplantation in China 1 month before CT examination, shows diffuse and mild dilatation of the peripheral intrahepatic bile ducts (arrows). The transplanted liver had been infected with $C$. sinensis. B. Periductal enhancement (arrows) with the thickened bile duct walls on hepatic arterial phase of dynamic study, which possibly represents active infection, in a 61-year-old man.

findings of clonorchiasis (Lim, 1990), however, it is also observed in cured infection.

In contrast to sonography showing an increased echogenicity of the ductal wall, CT failed to show thickening of the bile duct wall (Choi et al., 1989). A recent experimental study, however, suggested that an active infection could be differentiated from cured clonorchiasis by using the periductal enhancement with the thickened bile duct walls on dynamic contrast-enhanced CT (Lee et al., 2003). The contrast-
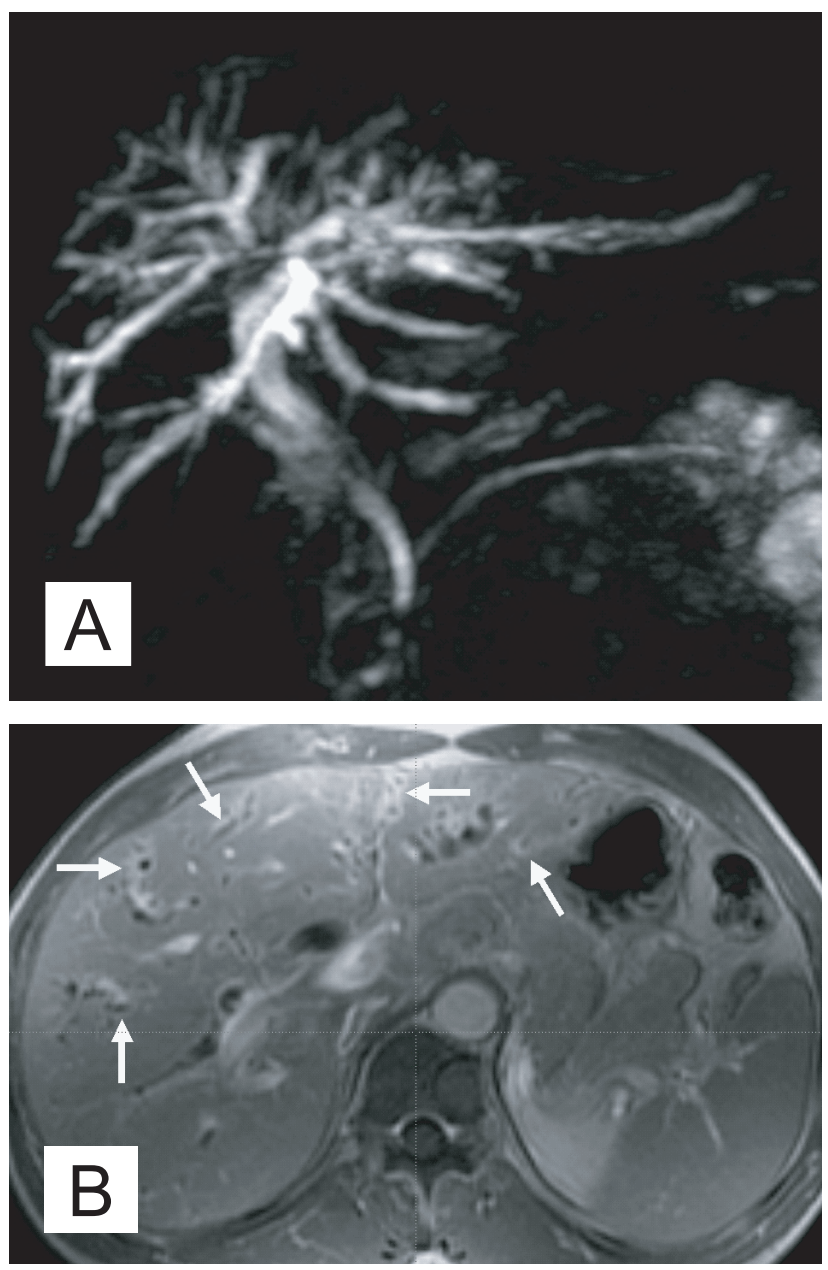

Fig. 4. MR findings of the liver in clonorchiasis patients. A. MR cholangiography in a 58-year-old man, who underwent cholecystectomy 10 year ago, showing diffuse and uniform dilatation of the peripheral intrahepatic bile ducts. B. Periductal enhancement of mildly dilated intrahepatic bile ducts (arrows) on hepatic arterial phase of dynamic study of gadolinium-enhanced echo fast gradient echo 3D (TR/TE, 7/2) transverse image, which possibly represents active infection, in a 60-year-old man (Courtesy of Dr. Yong Yeon Jeong, Gwangju, Korea).

enhanced CT visualized a characteristic finding, transient hepatic attenuation differences (THAD). The finding was interpreted as an outcome of new vascularization in the thickened bile duct wall, which disappeared after cure of clonorchiasis (Fig. 3B). They also asserted that some adult worms were demonstrated on their thin-section CT scans (Lee et al., 2003). 


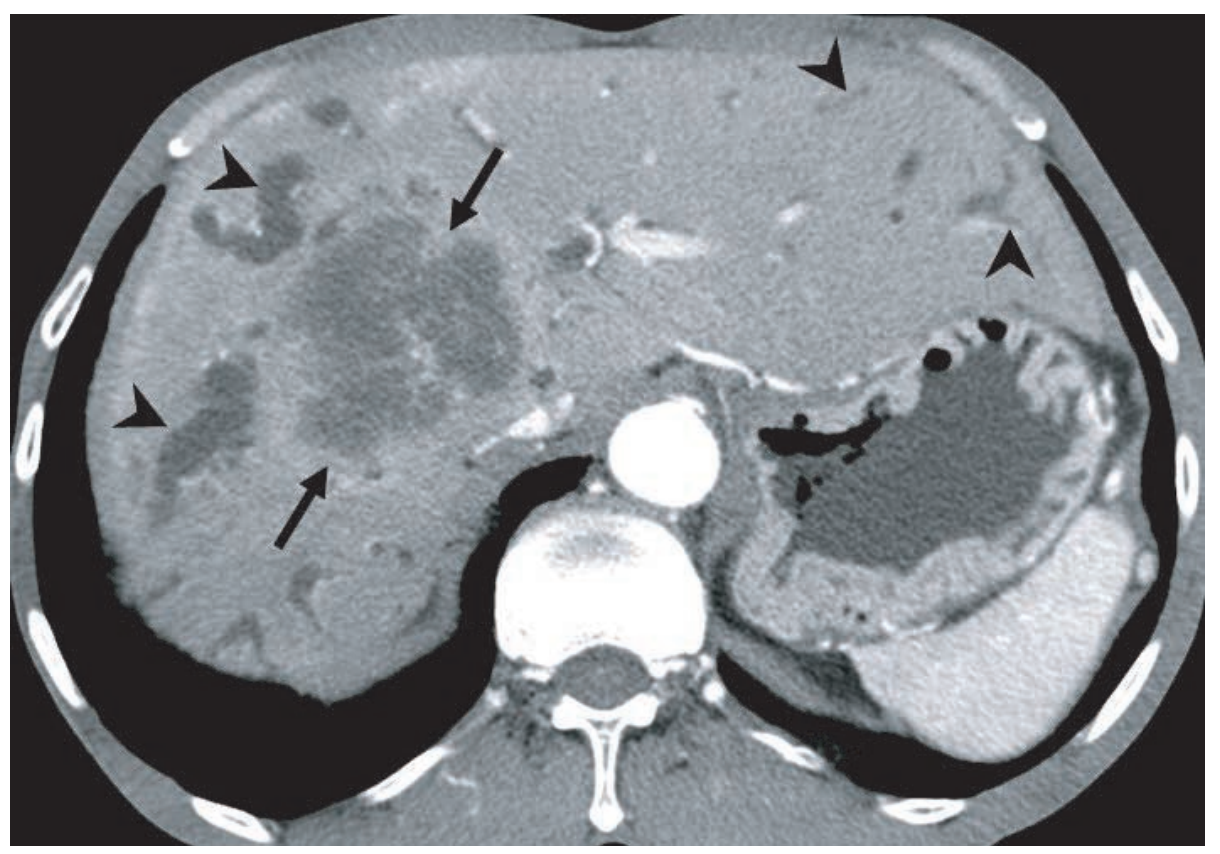

Fig. 5. Cholangiocarcinoma in the right hepatic lobe, with clonorchiasis, in a 63-year-old man. There is diffuse dilatation of peripheral intrahepatic bile ducts (arrowheads) is attributed to changes of a C. sinensis infection, and segmental and more severe dilatation around the tumor is caused by obstruction by the tumor (arrows).

\section{MR IMAGING FINDINGS OF CLONORCHIASIS}

MR imaging has been used in diagnosis of various diseases of the biliary system, and MR cholangiography is a relatively new non-invasive imaging modality for evaluation of the biliary pathology. MR imaging can show the characteristic findings of clonorchiasis with diffuse, mild dilation of the small intrahepatic bile ducts, particularly in the periphery of the liver (Choi et al., 1998). According to a recent study, MR cholangiography also found "too many intrahepatic ducts" sign in 62\% (16 of 26) of patients with clonorchiasis (Jeong et al., 2004). Periductal enhancement was more frequently seen on MR imaging, compared to CT, due to a higher spatial resolution of the soft tissue (Fig. 4). The filling defects representing worms were elliptical, irregular shaped, and hypointense lesions on the T2-weighted images and MR cholangiography.

\section{IMAGING FINDINGS OF CHOLANGIOCARCINOMA ASSOCIATED WITH CLONORCHIASIS}

The International Agency for Research on Cancer working group (1994) suggested that C. sinensis is a presumed carcinogen. Now, the relationship between clonorchiasis and cholangiocarcinomas is generally accepted, as epidemiological, pathological and experimental evidences, indicating a positive correlation, have been accumulated (Belamaric, 1973; Shin et al., 1996; Lee et al., 1997; Choi et al., 2006; Lim et al., 2006).

The imaging findings of clonorchiasis-associated cholangiocarcinomas are combinations of the findings of the 2 diseases (Figs. 5 and 6) (Choi et al., 2004). Diffuse dilatation of peripheral intrahepatic ducts is attributed to changes that are secondary to clonorchiasis, and segmental and severe dilatation around the tumor is caused by obstruction by the tumor (Fig. 5) (Choi et al., 1988 \& 1989). Extrahepatic cholangiocarcinomas, associated with clonorchiasis, show diffuse dilatation of the peripheral and central intrahepatic bile ducts (Fig. 6). When an extrahepatic cholangiocar- 

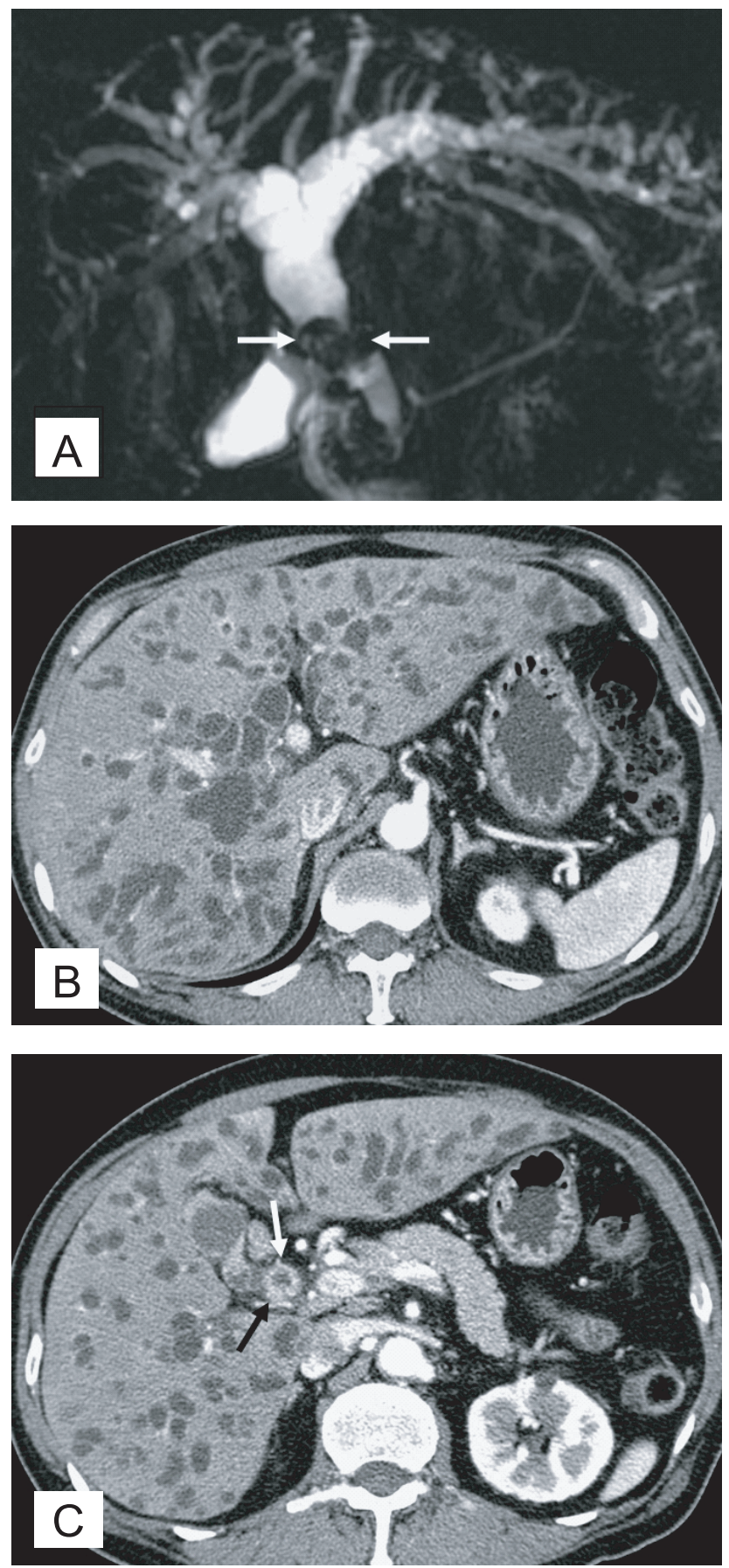

Fig. 6. Cholangiocarcinoma in the mid common duct, with clonorchiasis, in a 64-year-old man. A. MR cholangiography shows a diffuse dilatation of the intrahepatic bile ducts and common duct. Note an intraluminal tumor (arrows) at the level of the mid common duct. B. Contrastenhanced CT shows diffuse and marked dilatation of the intrahepatic bile ducts. C. CT at a level lower than (B) shows the enhancing, segmental thickened wall (arrows) of the mid common duct, which represents an extrahepatic cholangiocarcinoma.
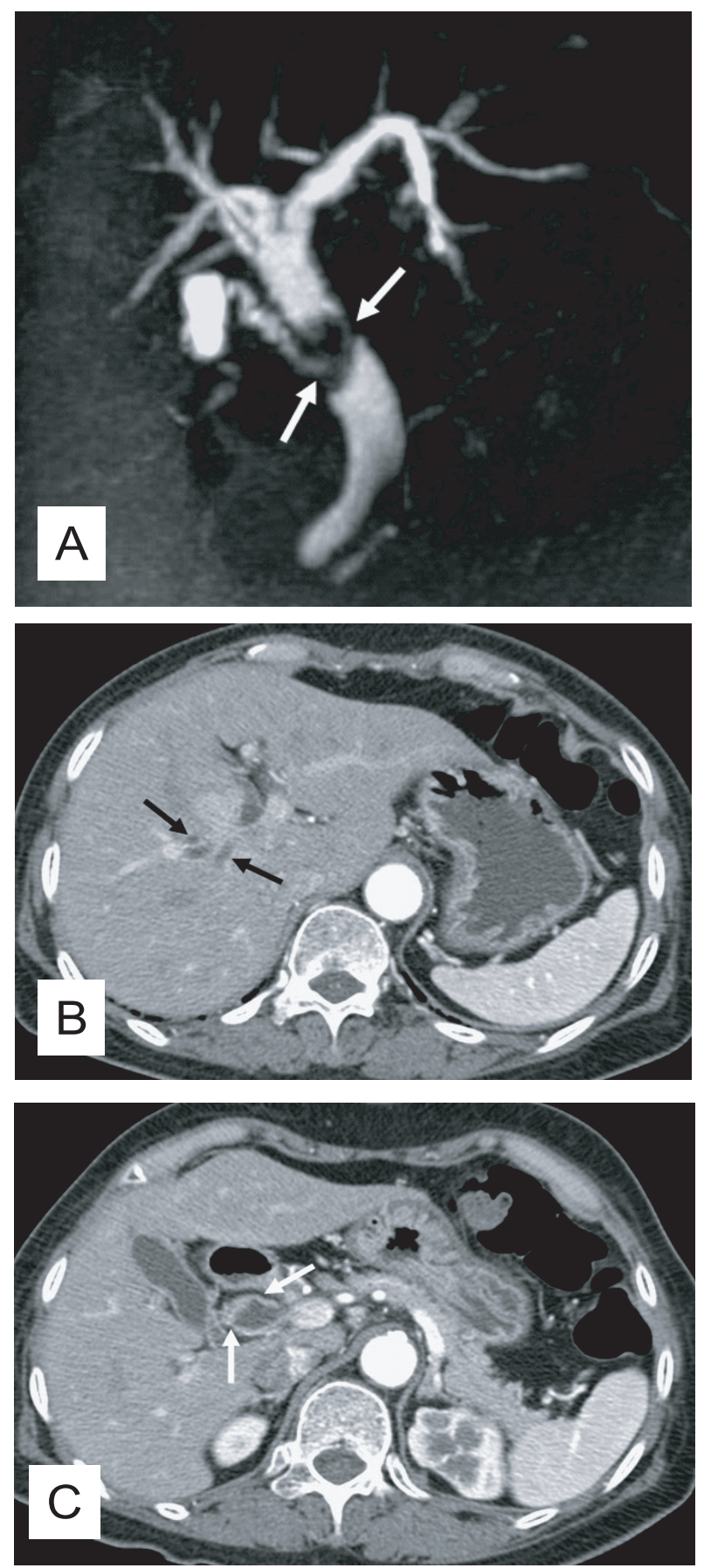

Fig. 7. Cholangiocarcinoma in the mid common duct, without clonorchiasis, in a 65-year-old woman. A. MR cholangiography shows an intraluminal tumor (arrows) at the level of the mid common duct. B. Contrast-enhanced CT shows minimal dilatation of the central intrahepatic duct (arrows), but the peripheral intrahepatic ducts are not dilated. C. CT at a level lower than (B) shows the enhancing, segmental thickened wall (arrows) of the mid common duct, which represents an extrahepatic cholangiocarcinoma. 
cinoma is not associated with clonorchiasis, dilatation of the peripheral intrahepatic bile ducts is not conspicuous (Fig. 7). After the development of the distal obstruction due to an extrahepatic cholangiocarcinoma, pre-existent dilated intrahepatic bile ducts with clonorchiasis easily progress the dilatation.

\section{DIFFERENTIAL DIAGNOSIS OF CLONORCHIASIS}

The differential diagnosis of clonorchiasis includes recurrent pyogenic cholangitis and primary sclerosing cholangitis. Recurrent pyogenic cholangitis is characterized by decreased branching and abrupt tapering of the peripheral hepatic ducts resulted from stenosis of the peripheral ducts (Lim, 1991; Kim et al., 1999). Dilatation of the extrahepatic bile duct and a stone on recurrent pyogenic cholangitis are more common than clonorchiasis. The pattern of intrahepatic bile duct dilation in clonorchiasis is diffuse, mild dilation of the intrahepatic bile duct without abrupt tapering of the peripheral hepatic ducts. However, because $C$. sinensis infection is one of the causes of recurrent pyogenic cholangitis, the mixed imaging findings of 2 disease entities are sometimes observed in a patient. Primary sclerosing cholangitis shows stenosis, beading and pruning of the dilated intrahepatic bile duct. However, the bile ducts in primary sclerosing cholangitis are much more irregular and serpiginous, which are unlikely in clonorchiasis (Ito et al., 1999). Of course, the primary sclerosing cholangitis is a very rare disease in endemic areas of clonorchiasis.

\section{CONCLUSIONS AND PERSPECTIVES}

Radiological examinations are essential in diagnosis of biliary tree diseases including clonorchiasis and cholangiocarcinomas. Clonorchiasis is primarily detected by screening sonography of the liver according to its pathognomonic findings of diffuse dilatation of the intrahepatic bile ducts, periductal echogenicity, and floating materials in the gallbladder. Periductal enhancement on dynamic contrast-enhanced CT or MR imaging may be a specific finding of active clonorchiasis. Imaging diagnosis is a good alternative method for diagnosis of clonorchiasis practically in endemic areas. Residents living in the endemic areas of clonorchiasis, who show images of diffuse dilatation of the intrahepatic bile ducts but have no history of specific medication, should be recommended to take praziquantel for treatment. For control of clonorchiasis in endemic areas, it is practically necessary to detect the infected residents by any diagnostic method, such as stool examination, serology, or radiological imaging.

\section{REFERENCES}

Barish MA, Soto JA (1997) MR cholangiopancreatography: technique and clinical applications. AJR Am J Roentgenol 169: 1295-1303.

Belamaric J (1973) Intrahepatic bile duct carcinoma and C. sinensis infection in Hong Kong. Cancer 31: 468-473.

Chen M, Lu Y, Hua X, Mott KE (1994) Progress in assessment of morbidity due to Clonorchis sinensis infection: a review of recent literature. Trop Dis Bull 91: R7-R65.

Choi BI, Kim HJ, Do YS, Han MH, Lee SH (1989) CT findings of clonorchiasis. AJR Am J Roentgenol 152: 281-284.

Choi BI, Kim TK, Han JK (1998) MRI of clonorchiasis and cholangiocarcinoma. JMRI 8: 359-366.

Choi BI, Park JH, Kim YI, Yu ES, Kim SH, Kim WH, Kim CY, Han MC (1988) Peripheral cholangiocarcinoma and clonorchiasis: CT findings. Radiology 169: 149-153.

Choi D, Hong ST, Lim JH, Cho SY, Rim HJ, Ji Z, Yuan R, Wang $S$ (2004) Sonographic findings of active Clonorchis sinensis infection. J Clin Ultrasound 32: 17-23.

Choi D, Lim JH, Hong ST (2004) Relation of cholangiocarcinomas to clonorchiasis and bile duct stones. Abdom Imaging 29: 590-597.

Choi D, Lim JH, Lee KT, Lee JK, Choi SH, Heo JS, Jang KT, Lee NY, Kim S, Hong ST (2006) Cholangiocarcinoma and Clonorchis sinensis infection: a case-control study in Korea. J Hepatol 44: 1066-1073.

Choi D, Lim JH, Kim SK, Kim EY, Lee M, Hong ST (1999) Long-lasting sonographic and histopathological findings in cured clonorchiasis of rabbits. Korean J Parasitol 37: 77-83.

Choi MS, Choi D, Choi MH, Ji Z, Li Z, Cho SY, Hong KS, Rim HJ, Hong ST (2005) Correlation between sonographic findings and infection intensity in clonorchiasis. Am J Trop Med Hyg 73: 1139-1144.

Choi TK, Wong KP, Wong J (1984) Cholangiographic 
appearance in clonorchiasis. Br J Radiol 57: 681-684.

Hong ST (2003) Clonorchis sinensis. In International handbook of foodborne pathogens, Miliotis MD, Bier JW (eds). pp 581-592, Marcel Dekker Inc, New York, USA.

Hong ST, Choi MH, Kim CH, Chung BS, Ji Z (2003) The Kato-Katz method is reliable for diagnosis of Clonorchis sinensis infection. Diagn Microbiol Infect Dis 47: 345-347.

Hong ST, Park KH, Seo M, Choi BI, Chai JY, Lee SH (1994) Correlation of sonographic findings with histopathological changes of the bile ducts in rabbits infected with Clonorchis sinensis. Korean J Parasitol 32: 223-230.

Hong ST, Yoon K, Lee M, Seo M, Choi MH, Sim JS, Choi BI, Yun CK, Lee SH (1998) Control of clonorchiasis by repeated praziquantel treatment and low diagnostic efficacy of sonography. Korean J Parasitol 36: 249-254.

International Agency for Research on Cancer working group (1994) Infection with liver flukes (Opisthorchis viverrini, Opisthorchis felinneus and Clonorchis sinensis). IARC Monogr Eval Carcinog Risks Hum 61: 121-175.

Ito K, Mitchell DB, Outwater EK, Blasbalg R (1999) Primary sclerosing cholangitis: MR imaging features. AJR Am J Roentgenol 172: 1527-1533.

Jeong YY, Kang HK, Kim JW, Yoon W, Chung TW, Ko SW (2004) MR imaging findings of clonorchiasis. Korean J Radiol 5: 25-30.

Kim MJ, Cha SW, Mitchell DG, Chung JJ, Park S, Chung JB (1999) MR imaging findings in recurrent pyogenic cholangitis. AJR Am J Roentgenol 173: 1545-1549.

Korea Association of Health Promotion (2004) Prevalence of intestinal parasitic infections in Korea - the 7th Report-. Seoul, Korea.

Lee JH, Rim HJ, Sell S (1997) Heterogeneity of the "ovalcell" response in the hamster liver during cholangiocarcinogenesis following Clonorchis sinensis infection and dimethylnitrosamine treatment. J Hepatol 26: 1313-1323.

Lee KH, Hong ST, Han JK, Yoon CJ, Lee S, Kim SH, Choi BI (2003) Experimental clonorchiasis in dogs: CT findings before and after treatment. Radiology 228: 131-138.

Lim JH (1990) Radiologic findings of clonorchiasis. AJR Am J Roentgenol 155: 1001-1008.

Lim JH (1991) Oriental cholangiohepatitis: pathologic, clinical, and radiologic features. AJR Am J Roentgenol 157: 18.

Lim JH, Ko YT, Lee DH, Kim SY (1989) Clonorchiasis: sonographic findings in 59 proved cases. AJR Am J Roentgenol 152: 761-764.

Lim JH, Ko YT, Lee DH, Lee KS, Suh SJ, Woo SK (1992) Clonorchiasis and its complications: cholangiogram revisited. J Korean Radiol Soc 28: 229-235.

Lim MK, Ju YH, Franceschi S, Oh JK, Kong HJ, Hwang SS, Park SK, Cho SI, Sohn WM, Kim DI, Yoo KY, Hong ST, Shin HR (2006) Clonorchis sinensis infection and increasing risk of cholangiocarcinoma in the Republic of Korea. Am J Trop Med Hyg 75: 93-96.

Shin HR, Lee CU, Park HY, Seol SY, Chung JM, Choi HC, Ahn YO, Shigemastu T (1996) Hepatitis B and C virus, Clonorchis sinensis for the risk of liver cancer: a case-control study in Pusan, Korea. Int J Epidemiol 25: 933-940. 
